\title{
Double quantum dots defined in bilayer graphene
}

\author{
D. P. Żebrowski, ${ }^{1}$ F. M. Peeters, ${ }^{2}$ and B. Szafran ${ }^{1}$ \\ ${ }^{1}$ Faculty of Physics and Applied Computer Science, AGH University of Science and Technology, aleja Mickiewicza 30, \\ 30-059 Kraków, Poland \\ ${ }^{2}$ Departement Fysica, Universiteit Antwerpen, Groenenborgerlaan 171, 2020 Antwerpen, Belgium
}

(Received 20 March 2017; revised manuscript received 19 June 2017; published 25 July 2017)

\begin{abstract}
Artificial molecular states of double quantum dots defined in bilayer graphene are studied with the atomistic tight-binding method and its low-energy continuum approximation. We indicate that the extended electron wave functions have opposite parities on sublattices of the layers and that the ground-state wave-function components change from bonding to antibonding with the interdot distance. In the weak-coupling limit, the one most relevant for quantum dots defined electrostatically, the signatures of the interdot coupling include, for the two-electron ground state, formation of states with symmetric or antisymmetric spatial wave functions split by the exchange energy. In the high-energy part of the spectrum the states with both electrons in the same dot are found with the splitting of energy levels corresponding to simultaneous tunneling of the electron pair from one dot to the other.
\end{abstract}

DOI: 10.1103/PhysRevB.96.035434

\section{INTRODUCTION}

Studies of artificial molecules formed by carrier orbitals extended over double quantum dots (DQDs) focus on carrier tunneling and interactions [1-5,7]. Particular attention is paid to the spin-related phenomena in the context of quantum information processing [8] using exchange interaction due to the interdot coupling [7,9]. The coupling with nuclear spins [10-13] which limits the electron-spin-coherence times motivated studies of DQDs with holes as spin carriers [14,15] as well as on systems based on silicon [16] and carbon, including nanotubes [17] and graphene [18]. In graphene the carrier storage is hampered by the Klein tunneling [19], but for nanoribbons the lateral confinement opens the transport gap [20] that makes the carrier storage possible [21]. Quantum dots in ribbons are influenced by edge effects and disorder [22]. An alternative medium is the bilayer graphene [23] for which the perpendicular electric field opens the band gap [24] in the energy spectrum and allows for carrier confinement by lateral fields [25-30].

In this work we consider bilayer graphene [23] and the formation of extended orbitals within the DQDs. In the lowenergy continuum approach Hamiltonian eigenstates possess a definite total angular momentum [25] with a different orbital angular momentum for each of the four sublattices described by the wave-function components.

As we discuss below for a lower point symmetry of the DQD system, the Hamiltonian eigenstate components correspond to opposite spatial parities for each of the sublattices. Thus the wave function can be bonding on one sublattice and antibonding on the other. The effect induces a complex dependence of the spectrum on the DQD distance that is similar to the antibonding heavy-hole ground state found for vertical self-assembled quantum dots [3-6].

In the low-energy part of the two-electron spectrum for the weak-coupling case, the typical one for electrostatic quantum dots, the electrons are localized in separate QDs, and the ground state at zero magnetic field $B$ is nearly 16 times degenerate with respect to the valley and the spin. For nonzero magnetic field the energy levels correspond to a wave function that can be approximately described by a product of the spatial, valley, and spin components. Only the symmetry of the spatial part against the electron interchange influences the energy of the states, and we find that the energy levels shift in pairs with $B$ that correspond to the opposite symmetry split by the exchange integral. In the high-energy part of the spectrum the states corresponding to both electrons in the same dot are found. These energy levels also shift in $B$ in pairs, which is a manifestation of collective two-electron interdot tunneling that forms bonding and antibonding two-electron orbitals. For stronger interdot coupling the DQD spectrum resembles that of a single quantum dot with a ground-state triplet [31].

This paper is organized as follows. In Sec. II we describe the model system, the atomistic and continuum approaches, and the configuration-interaction method for the electron pair. In Sec. III we discuss the formation of the extended orbitals and their nature as artificial molecular states, and then we discuss the spatial, spin, and orbital symmetries of the two-electron states. A summary and conclusions are given in Sec. IV.

\section{THEORY}

\section{A. Model structure}

We consider a flake in the form of a stretched hexagon (see Fig. 1) with the bilayers in Bernal stacking [23] and two quantum dots defined by the external potential. We consider two sizes of flakes. The smaller flake has a length of $L=$ $64.6 \mathrm{~nm}$ and height $h=27.6 \mathrm{~nm}$, with a horizontal edge length of $L^{\prime}=48.8 \mathrm{~nm}$, and the total number of carbon atoms is 119 928. The larger flake is characterized by $L=89.46 \mathrm{~nm}$, $L^{\prime}=63.9 \mathrm{~nm}$, and $h=44.51 \mathrm{~nm}$ and contains 261000 atoms. Two different sizes of flakes for a fixed external potential defining the quantum dots are introduced for the discussion of the effects of the coupling between the dot-confined states and the edge of the flake.

We consider a flake with an armchair boundary for which no edge-localized energy levels appear in the spectrum at the neutrality point $[30,32,33]$. The quantum dots for electrons of the conduction band are defined by the external potential which takes the form

$$
V_{Q D}(x, y)=-V \exp \left\{-\left[(|x|-d)^{2}+y^{2}\right] / R^{2}\right\}
$$




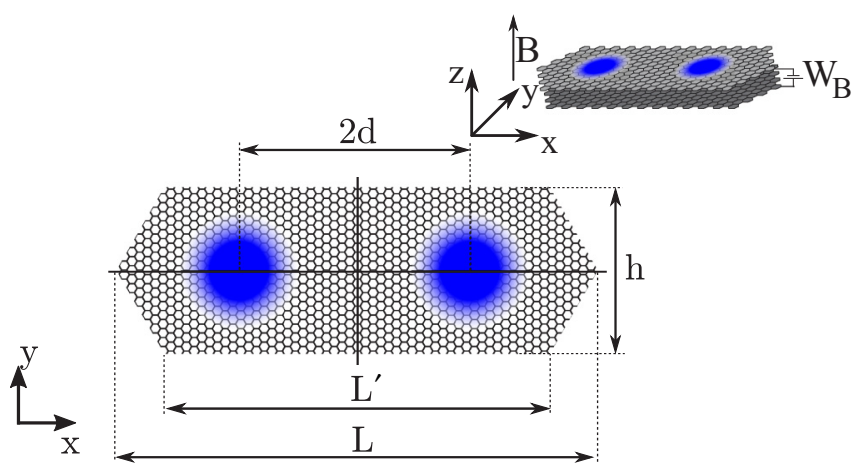

FIG. 1. Schematic drawing of the considered system. A bilayer graphene flake in a hexagonal shape is stretched in the $x$ direction with quantum dots separated by $2 d$ represented by blue circles. We consider flakes of two sizes. The length of the smaller system is $L=64.6 \mathrm{~nm}$, the height is $h=27.6 \mathrm{~nm}$, and the longer edge has a length of $L^{\prime}=48.8 \mathrm{~nm}$; the parameters for the larger system are $L=89.46 \mathrm{~nm}, L^{\prime}=63.9 \mathrm{~nm}$, and $h=44.51 \mathrm{~nm}$.

where the origin $(x=y=0)$ is placed in the center of the flake, $V$ is the quantum dot depth, $R$ is the effective radius, and $2 d$ is the distance between the centers of the QDs. In this work, we fix $R$ at $4 \mathrm{~nm}$; for this value the wave functions localized in the dots vanish off the edges of the larger flake, for which the valley mixing effect of the armchair edge is negligible. For the smaller flake the effect of the edge for the confined states is still present, and we use this fact below for the description of the valley mixing effects on the single- and the two-electron spectra.

\section{B. Tight-binding model}

For most of the calculations we use the atomistic tightbinding Hamiltonian [23] given by

$$
\hat{H}_{1 e}=\sum_{i, \sigma} W_{i}^{\sigma} \hat{c}_{i, \sigma}^{\dagger} \hat{c}_{i, \sigma}+\sum_{i j, \sigma \sigma^{\prime}}\left(\hat{c}_{i, \sigma}^{\dagger} \hat{c}_{j, \sigma^{\prime}} t_{i j}+\text { H.c. }\right),
$$

where $\hat{c}_{i, \sigma}^{\dagger}\left(\hat{c}_{i, \sigma}\right)$ creates (annihilates) an electron with spin $\sigma$ at ion $i$ and $t_{i j}$ is the hopping parameter. At zero magnetic field the hopping parameter takes the value $t_{i j}=-2.6 \mathrm{eV}$ for the in-plane nearest-neighbor atoms and $t_{i j}=0.3 \mathrm{eV}$ for the vertical dimers between the layers [23]. In order to account for the magnetic field perpendicular to the layers we include the Peierls phase in the hopping parameters,

$$
t_{i j} \rightarrow t_{i j} e^{i \frac{e}{\hbar} \int_{\mathbf{r i}_{\mathbf{i}}}^{\mathbf{r}_{\mathbf{j}}} \mathbf{A} \cdot \mathbf{d l} \mathbf{l}},
$$

where $\mathbf{B}=\nabla \times \mathbf{A}$, with $\mathbf{A}=\left(0, B_{z} x, 0\right)$. The potential $W_{i}^{\sigma}$ in Eq. (2) has the form

$$
W^{\sigma}(x, y)=V_{Q D}(x, y)+\frac{W_{B}}{2} \tau_{z}+\frac{1}{2} \mu_{B} g \sigma_{z} B_{z},
$$

where the first term is given by Eq. (1) and the second one is the potential difference between the layers with $\tau_{z}= \pm 1$, with $+(-)$ for the upper (lower) layer. This difference opens the energy gap in the bilayer flake by the asymmetry introduced between the layers [23]. In our calculations we set $W_{B}=$ $300 \mathrm{meV}$, which is equivalent to applying the vertical electric field $F \approx 0.9 \mathrm{~V} / \mathrm{nm}$. For this value we obtain a gap of about
$E_{g} \approx 141 \mathrm{meV}$. The last term in Eq. (4) introduces the Zeeman interaction, where $g=2$ is the Landé factor and $\sigma_{z}$ stands for the Pauli matrix.

\section{Continuum approximation}

For analysis of the single-electron envelope wave functions, we also consider the low-energy approximation in the continuum model. Near a single valley the electron wave functions are written in the form of the four-component spinor [23],

$$
\Psi(\mathbf{r})=\left(\phi_{A}(\mathbf{r}), \phi_{B}(\mathbf{r}), \phi_{B^{\prime}}(\mathbf{r}), \phi_{A^{\prime}}(\mathbf{r})\right)^{T},
$$

where $A$ and $B$ ( $B^{\prime}$ and $\left.A^{\prime}\right)$ represent the $A$ and $B$ sublattices of the upper (lower) layer, respectively. The Dirac Hamiltonian [23] around the $\mathbf{K}$ valley takes the form

$$
\hat{H}_{D}=\left(\begin{array}{cccc}
V_{Q D} & \pi & t_{\perp} & 0 \\
\pi^{\dagger} & V_{Q D} & 0 & 0 \\
t_{\perp} & 0 & V_{Q D} & \pi^{\dagger} \\
0 & 0 & \pi & V_{Q D}
\end{array}\right)+\frac{W_{B}}{2} \tau_{z},
$$

where $\pi=v_{F}\left(p_{x}+i p_{y}\right),\left(p_{x}, p_{y}\right)$ is the momentum operator, $v_{F}=\frac{3 t a}{2}=0.84 \times 10^{6} \frac{\mathrm{m}}{\mathrm{s}}$ is the Fermi velocity, and $t_{\perp}=$ $0.3 \mathrm{eV}$ is the interlayer coupling term. The $\tau_{z}$ operator defined as

$$
\tau_{z}=\left(\begin{array}{cc}
\mathbb{I}_{2} & 0 \\
0 & -\mathbb{I}_{2}
\end{array}\right),
$$

which assigns $+1(-1)$ for the upper (lower) layer, opens the gap by applying the voltage difference $W_{B}$ between the layers.

The quantum dot potential (1) is symmetric with respect to the point inversion at the origin. For this potential the Hamiltonian commutes with a generalized parity operator

$$
\hat{U}_{P}=\left(\begin{array}{cc}
-\sigma_{z} & 0 \\
0 & -\sigma_{z}
\end{array}\right) \hat{P},
$$

where $\hat{P}$ changes the sign of the spatial coordinates of scalar functions $\hat{P} \phi(\mathbf{r})= \pm \phi(-\mathbf{r})$ and $\mathbb{I}_{n}$ is the $n \times n$ identity matrix.

For a single circular quantum dot the total angular momentum operator

$$
\hat{J}_{z}=\hat{L}_{z} \mathbb{I}_{4}+\frac{\hbar}{2} \tau_{z}-\frac{\hbar}{2}\left(\begin{array}{cc}
\sigma_{z} & 0 \\
0 & -\sigma_{z}
\end{array}\right)
$$

commutes with the Hamiltonian [25], where $\hat{L}_{z}$ is the angular momentum operator. The second term is the layer index operator with $\tau_{z}$ defined by (7). The last term corresponds to the pseudospin [23].

In order to find the eigenstates of the Hamiltonian (6) we use the finite-element method with triangular elements and cubic shape functions. The fermion-doubling fast-varying spurious solutions [34] are eliminated with the additional Wilson [34] term introduced to the Hamiltonian (6),

$$
\hat{H}_{W}=W_{P} \hbar v_{F} a_{D}\left(\begin{array}{cc}
-\sigma_{z} & 0 \\
0 & -\sigma_{z}
\end{array}\right) \nabla^{2},
$$

where $W_{P}=0.015$ is the dimensionless Wilson parameter [34] and $a_{D}=1.32 \mathrm{~nm}$ is the discretization constant. The $W_{P}$ parameter was fine-tuned to remove the spurious solutions from the discussed energy range, leaving the actual states nearly unaffected. 


\section{Configuration-interaction method}

The wave functions obtained with the tight-binding approach are used in the configuration-interaction method $[35,36]$. The atomistic approach when applied to the exact diagonalization method naturally accounts for the intervalley scattering induced by the short-range component of the Coulomb interaction [37-39].

For the description of the two-electron states we use the solution of the one-electron eigenproblem (2). Then, we expand the two-electron wave function in the basis of $M$ Slater determinants,

$$
\Psi=\sum_{i=1}^{M} d_{i}\left[\psi_{i 1}\left(\mathbf{x}_{1}\right) \otimes \psi_{i 2}\left(\mathbf{x}_{\mathbf{2}}\right)-\psi_{i 2}\left(\mathbf{x}_{\mathbf{1}}\right) \otimes \psi_{i 1}\left(\mathbf{x}_{\mathbf{2}}\right)\right],
$$

with spin orbitals $\psi_{i}(\mathbf{x}), i=1,2, \ldots, M, i_{1}, i_{2} \in[1, K]$, where $\mathbf{x}=(\mathbf{r}, \sigma)$ represents the orbital and spin coordinates. The number of Slater determinants $M$ is $\left(\begin{array}{c}K \\ 2\end{array}\right)$, where $K$ is the number of dot-localized single-electron spin orbitals. The two-electron Hamiltonian is

$$
\hat{H}_{2 e}\left(\mathbf{x}_{\mathbf{1}}, \mathbf{x}_{\mathbf{2}}\right)=\sum_{i=1}^{2} \hat{H}_{1 e}\left(\mathbf{x}_{\mathbf{i}}\right)+\frac{\kappa}{\left|\mathbf{r}_{\mathbf{1 2}}\right|},
$$

where $\kappa=e^{2} /\left(4 \pi \epsilon \epsilon_{0}\right)$. The dielectric constant $\epsilon=6$ is taken from Ref. [40] for graphene grown on SiC. Using the form of the wave function given by (11), we arrive at the Hamiltonian of the form

$$
\hat{H}_{2 e}=\sum_{i j} \hat{d}_{i}^{\dagger}\left\langle\psi_{i} \mid \hat{H} \psi_{j}\right\rangle \hat{d}_{j}+\frac{1}{2} \sum_{i j k l} \hat{d}_{i}^{\dagger} \hat{d}_{j}^{\dagger} \hat{d}_{k} \hat{d}_{l} V_{i j k l},
$$

where $\hat{d}_{i}^{\dagger}$ creates an electron in the $i$ th spin orbital. The oneelectron energy is taken into account with the matrix elements $\left\langle\psi_{i} \mid \hat{H} \psi_{j}\right\rangle$. The Coulomb matrix element

$$
V_{i j k l}=\kappa\left\langle\psi_{i}\left(\mathbf{x}_{\mathbf{1}}\right) \psi_{j}\left(\mathbf{x}_{\mathbf{2}}\right) \frac{1}{\left|\mathbf{r}_{\mathbf{1 2}}\right|} \psi_{k}\left(\mathbf{x}_{\mathbf{1}}\right) \psi_{l}\left(\mathbf{x}_{\mathbf{2}}\right)\right\rangle
$$

for the single-electron wave functions given by linear combinations of atomic orbitals $p_{z}$ gives

$$
\begin{aligned}
V_{i j k l}= & \kappa\left\langle\psi_{i}\left(\mathbf{x}_{\mathbf{1}}\right) \psi_{j}\left(\mathbf{x}_{\mathbf{2}}\right)\left|\frac{1}{\left|\mathbf{r}_{\mathbf{1 2}}\right|}\right| \psi_{k}\left(\mathbf{x}_{\mathbf{1}}\right) \psi_{l}\left(\mathbf{x}_{\mathbf{2}}\right)\right\rangle \\
= & \kappa \sum_{\substack{a, \sigma_{a} ; b, \sigma_{b} ; \\
c, \sigma_{c} ; d, \sigma_{d}}} \beta_{a, \sigma_{a}}^{i *} \beta_{b, \sigma_{b}}^{j *} \beta_{c, \sigma_{c}}^{k} \beta_{d, \sigma_{d}}^{l} \delta_{\sigma_{a} ; \sigma_{d}} \delta_{\sigma_{b} ; \sigma_{c}} \\
& \times\left\langle p_{z}^{a}\left(\mathbf{r}_{1}\right) p_{z}^{b}\left(\mathbf{r}_{\mathbf{2}}\right)\left|\frac{1}{\left|\mathbf{r}_{\mathbf{1 2}}\right|}\right| p_{z}^{c}\left(\mathbf{r}_{\mathbf{1}}\right) p_{z}^{d}\left(\mathbf{r}_{\mathbf{2}}\right)\right\rangle .
\end{aligned}
$$

For the Coulomb integral we apply the two-center approximation [41] $\left\langle p_{z}^{a}\left(\mathbf{r}_{1}\right) p_{z}^{b}\left(\mathbf{r}_{2}\right)\left|\frac{1}{\left|\mathbf{r}_{12}\right|}\right| p_{z}^{c}\left(\mathbf{r}_{1}\right) p_{z}^{d}\left(\mathbf{r}_{2}\right)\right\rangle=\frac{1}{r_{a b}} \delta_{a c} \delta_{b d}$ for $a \neq b$, and for the single-center integral $a=b$ we take $16.522 \mathrm{eV}$ [36].

\section{RESULTS}

\section{A. Single-electron energy levels}

The single-electron spectrum obtained with the atomistic tight-binding method is displayed in Fig. 2 as a function of the depth of the dots for the interdot distance $2 d=20 \mathrm{~nm}$ for zero

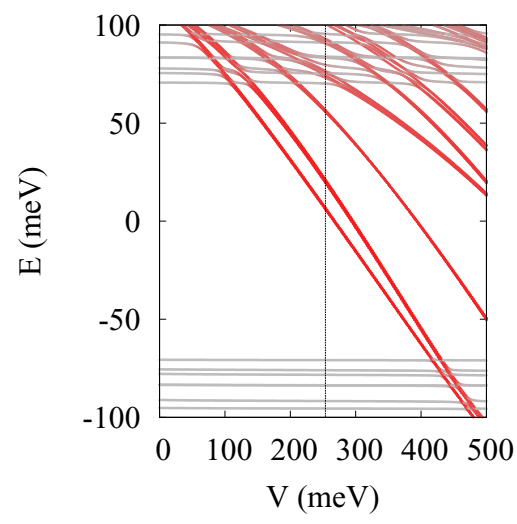

FIG. 2. The energy spectrum as a function of the quantum dot depth $V$ for an interdot distance of $2 d=20 \mathrm{~nm}$ at zero magnetic field. The red (gray) curves indicate the localization of electron density inside (outside) the quantum dots. The dashed line shows the potential depth chosen for further calculations.

magnetic field. The color of the lines indicates the extent of the electron localization within QDs: the red ones are localized in the QD potentials, and the gray energy levels correspond to the states which are localized outside the dots. Figure 2 shows that the latter ignore the QD potential. The vertical line in Fig. 2 indicates the potential value $V=250 \mathrm{meV}$ that is taken for further calculations. Each of the energy levels plotted in Fig. 2 is nearly fourfold degenerate with respect to the spin and valley.

For identical quantum dots the splitting between the two lowest energy levels is a result of the interdot coupling. These energy levels tend to a further degeneracy at large $d$ (see Fig. 3). Typically, in coupled quantum dots for small $d$ the bonding and antibonding electron orbitals are formed, and the splitting energy in III-V systems is a monotonic function of $d$ [9]. That is not the case that we observe in Fig. 3(a), where crossings of the two lowest energy levels are seen.

The energy spectrum obtained with the continuum approximation of Figs. 3(c) and 3(d) is similar to the exact results of Figs. 3(a) and 3(b). In Fig. 3(c) we plot with the color of the line the average value of the total angular momentum (9) calculated for the envelope function of the continuum Hamiltonian. At large $d$, where the interdot tunneling is negligible, and for $d=0$, where the potential has a rotational symmetry, the $\hat{J}_{z}$ eigenvalue is a good quantum number. The separate components of the envelope wave function correspond then to the angular momentum quantum number [25] of $(m, m+1, m, m-1)$ for sublattices $A, B, B^{\prime}$, and $A^{\prime}$, respectively. The ground state for the circular QD corresponds to $m=1$, and the lowest excited state corresponds to $m=0$, in accordance with Ref. [25].

For a general interdot distance $d$ the total angular momentum is no longer quantized. However, each of the components has strict symmetry with respect to the point inversion through the center between the dots. The total wave function is an eigenstate of $\hat{U}_{P}$, and the eigenvalues \pm 1 are marked in Fig. 3(d) with the color of the line.

In order to analyze in more detail the formation of the extended orbitals by the single-dot wave functions we constructed the localized (ionic) orbitals of the left $l$ and 


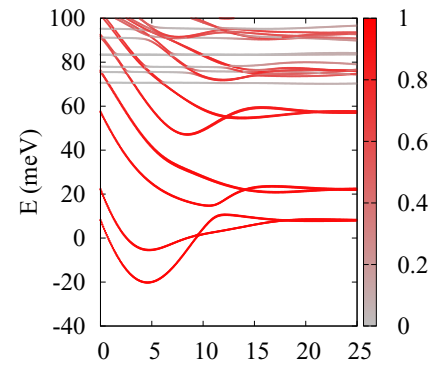

(a)
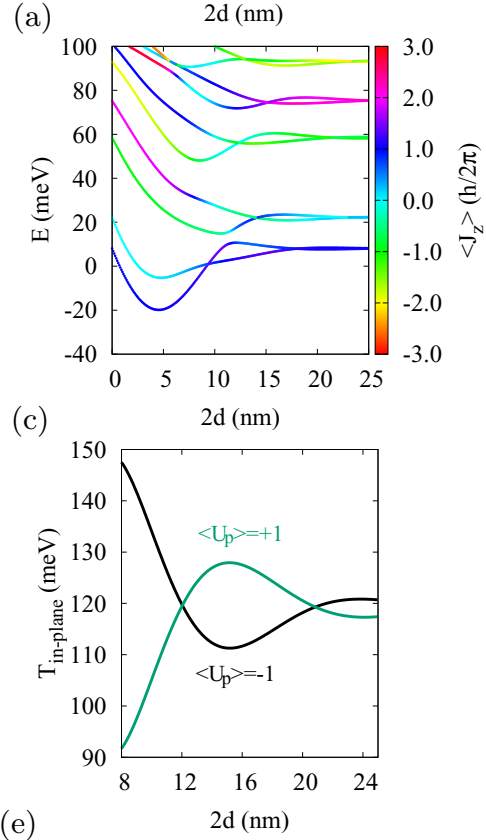

(e)

FIG. 3. Single-electron spectrum as a function of the interdot distance as obtained (a) and (b) with the atomistic tight-binding method and (c) and (d) with the low-energy continuum approximation. (a) The color of the lines indicates the electron localization, with the charge density integrated within the distance of $2 R$ from the centers of the dots. (b) Zoom of (a). The red lines are the same as in (a), while the orange and blue ones have been obtained as the sum or difference of the wave functions localized in single dots (see text). (c) The energy levels obtained with the low-energy approximation, with the color indicating the average value of the total angular momentum (9). (d) Same as (c), but with the color of the line indicating the eigenvalue of the $\hat{U}_{P}$ operator: the green one is +1 , and the black one is -1 . (e) The in-plane kinetic energy for the lowest-energy $\hat{U}_{P}$ eigenstates, showing the average value of the off-diagonal part of Hamiltonian (6) with $t_{\perp}$ excluded. (f) The kinetic energy for the $\hat{U}_{P}$ eigenstates with both intralayer $(\pi)$ and interlayer $\left(t_{\perp}\right)$ hopping, showing the average value of the entire off-diagonal part of Hamiltonian (6).

right $r$ quantum dots using the tight-binding approach. The wave functions $l$ and $r$ were constructed separately from (1) the two lowest energy states $\phi_{1}$ and $\phi_{2}$ and (2) the third and fourth states $\phi_{3}$ and $\phi_{4}$. The ionic functions were taken as a superposition of the Hamiltonian eigenstates, $l_{1,2}=$ $\left[\phi_{1}+\exp (i \alpha) \phi_{2}\right], r_{1,2}=\left\{\phi_{1}+\exp [i(\alpha+\pi)] \phi_{2}\right\}$, where the phase $\alpha$ was taken to maximize the electron localization at the left or right side of the origin. Then for Fig. 3(b) the extended states were produced as constructive and destructive interference of the $l$ and $r$ functions, $\phi_{c}^{1,2}=\frac{1}{\sqrt{2}}\left(l_{1,2}+r_{1,2}\right)$ and $\phi_{d}^{1,2}=\frac{1}{\sqrt{2}}\left(l_{1,2}-r_{1,2}\right)$, respectively. A similar operation was performed starting from the third and fourth energy-level wave functions $\phi_{3}$ and $\phi_{4}$. The mean values of the energy calculated for $\phi_{c}$ and $\phi_{d}$ wave functions are plotted by blue and orange lines in Fig. 3(b), respectively. The red line shows the exact result, which is the same as in Fig. 3(a). Note that there is a one-to-one correspondence between the $\hat{U}_{P}$ eigenvalue and the superposition sign taken in Fig. 3(b).

For the scalar electron envelope function in a III-V material $\phi_{c}$ and $\phi_{d}$ would correspond simply to the bonding and antibonding orbitals. For multicomponent wave functions of opposite symmetry the situation is more complex. In particular, for holes in the artificial molecules formed by vertical quantum dots [3-6] the wave functions need to be described by multicomponent wave functions for each of the valence bands that become degenerate at the $\Gamma$ point. In these systems the parity of the heavy-hole component is opposite the light hole component. For large interdot distances the interdot tunneling is carried by the light-hole component, and a bonding state at the light-hole component is formed, which triggers an antibonding heavy-hole ground state [3-6]. In III-V materials the crossing of the states of opposite parities is observed only for the vertical coupling [3-5] and not the lateral coupling of the quantum dots [6].

For the lateral DQD studied here the origin of the nonmonotonic behavior of the spacing between the lowest energy levels in Figs. 3(c) and 3(d) can be understood by inspecting the wave function. In Fig. 4 we plot the real part of the wave functions for energy levels and $d$ values marked by the corresponding letters in Fig. 3(d). For the plots at large interdot distances [see Figs. 4(c), 4(e) and 4(f)], each of the wave-function components depends on the azimuthal angle as $\operatorname{Re}[\exp (i L \Phi)]$ like for the angular momentum z-component eigenfunctions with quantum number $L$. In particular, in the lowest-energy state that is twofold degenerate at large $d$ [see Figs. 4(e) and 4(f)] the total angular momentum quantum number is $m=1$, and the $\hat{U}_{P}$ eigenvalue is +1 in Fig. 4(e) and -1 in Fig. 4(f). As the interdot distance is varied [see Fig. 3(d)], the energies of the states change but in a complex manner since two components of the wave functions are even in terms of the $\hat{U}_{P}$ operator, while the other two are odd, and the two parities have opposite consequences for energies as $d$ is modified. Moreover, the contributions of the components change. For instance, the state with the wave function given in Fig. 4(f) has a dominant $A^{\prime}$ component which is antibonding. For lower $d$ the $A$ and $B$ components [Fig. 4(d)] are increased at the expense of $A^{\prime}$ one. The black energy level in Fig. 3(d) becomes the ground-state level between interdot distances of 8 and $16 \mathrm{~nm}$. The varied contributions of the components involve avoided crossings between the energy levels of the same $\hat{U}_{P}$ parity, and the identity of the lines that avoided crossing can be traced by the average angular momentum [Fig. 3(c)]. At $d=0$ the sequence of $J_{z}$ eigenvalues in the order of the energy is the same as in the large $d$ limit.

In order to analyze the effect of the molecular coupling on the energy levels and the crossing observed in Figs. 3(a)-3(d) we plot in Fig. 3(e) the contribution of the in-plane kinetic 


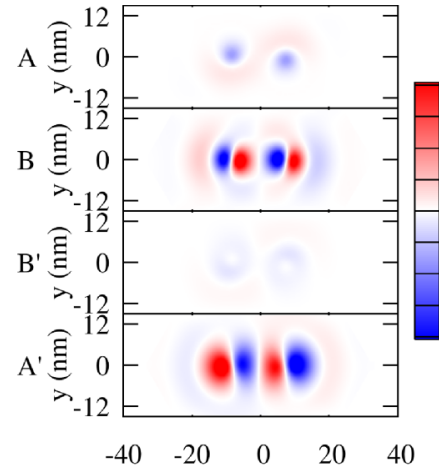

(a)

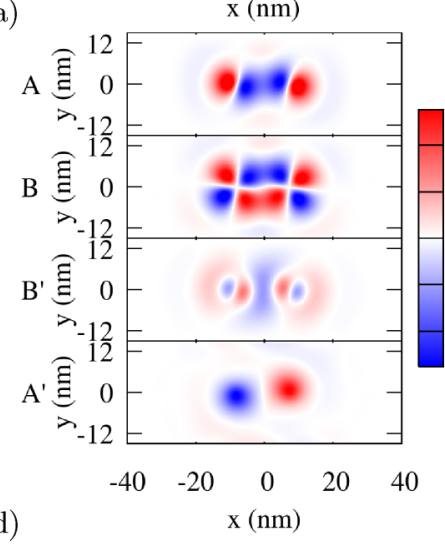

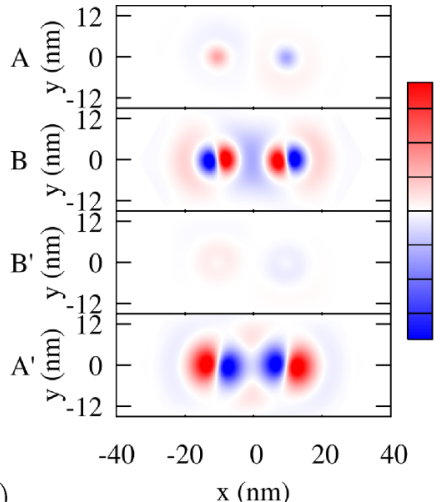

(b)

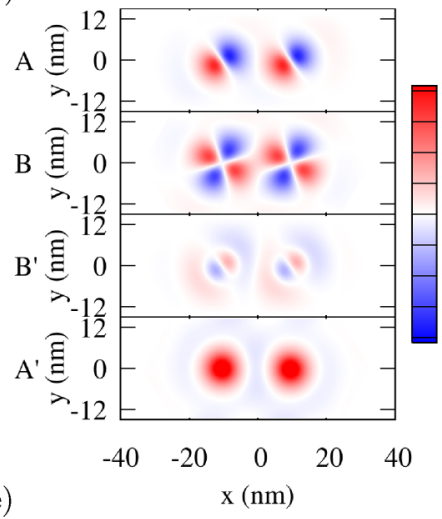

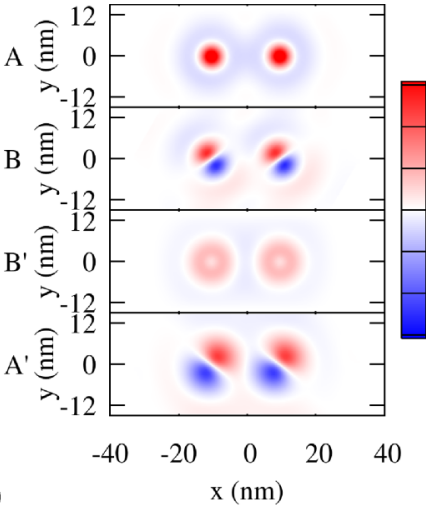

(c)

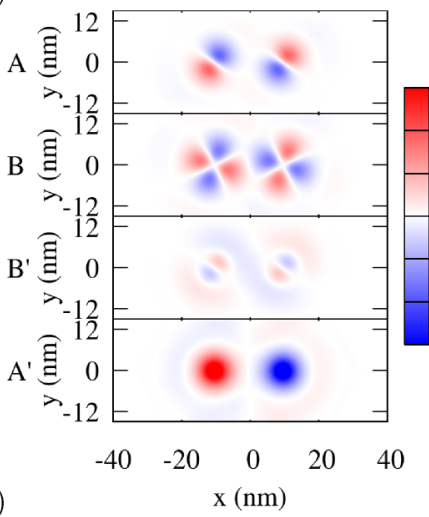

FIG. 4. Real part of the wave functions for the energy levels indicated by the corresponding letters in Fig. 3(d) at the sublattices of both layers. The states labeled (a) and (d) correspond to $2 d=8 \mathrm{~nm}$, and the rest of the plots correspond to $2 d=20 \mathrm{~nm}$. The deepest red (blue) color indicates the most positive (negative) value of the real part of the wave function, and white indicates its vanishing value.

energy for hopping between $A-B$ and $A^{\prime}-B^{\prime}$ sublattices within each layer that is due to the $\pi$ operator in the Hamiltonian given by Eq. (6). Figures 4(d), 4(e) and 4(f) indicate that for the ground state the coupling of the single-dot energy levels, each corresponding to $m=1$, appears for the largest interdot distances at the $B$ component which corresponds to orbital angular momentum quantum number $L=m+1=2$. The state that forms a bonding component there [Fig. 4(e)] with a positive eigenvalue of the $\hat{U}_{P}$ operator decreases in energy [Fig. 3(e)] due to the interdot coupling. When the dots get closer, the components with orbital angular momentum $L=m=1$ at the $A$ and $B^{\prime}$ sublattices form a molecular orbital which is antibonding (bonding) for the $\hat{U}_{P}$ eigenstate with an eigenvalue of $+1(-1)$. Due to the inverse contribution to the energy the first crossing at the ground-state energy level is observed near $2 d=18 \mathrm{~nm}$ [Figs. 3(a) and 3(d)].

The in-plane kinetic energies for the two states cross for an even larger distance [Fig. 3(e)] of $2 d=21 \mathrm{~nm}$. For the interlayer hopping between the $A$ and $B^{\prime}$ sublattices included, the crossing of kinetic energies occurs closer [Fig. 3(f)] to the intersection of the energy levels [Fig. 3(d)].

The second crossing of the ground-state energy levels near $2 d=8 \mathrm{~nm}$ results from the activation of the interdot tunnel coupling for the $A^{\prime}$ component of the angular momentum $L=m-1=0$ [Figs. 4(d)-4(f)]. In conclusion, the groundstate energy-level crossings for states with opposite $\hat{U}_{P}$ parity result from the subsequent switching of the interdot tunnel coupling for separate components of the wave function at the sublattices.
The magnetic field dependence of the energy spectrum is given in Fig. 5. At this energy scale the results for the smaller [Fig. 5(a)] and larger [Fig. 5(b)] flakes become distinguishable. For the smaller flake we notice avoided crossings between the energy levels of opposite valleys which result from the valley mixing effect of the armchair edge of the flake (at $B \simeq 0$ and $B \simeq 0.4 \mathrm{~T}$ ). The valley mixing opens an avoided crossing between the states of the same spin and $\hat{U}^{P}$ eigenvalue, and outside of the avoided crossings the energy levels in Fig. 5(a) can be attributed to the valley and spin quantum numbers. For the larger flake the valley mixing effects are not resolved on this scale.
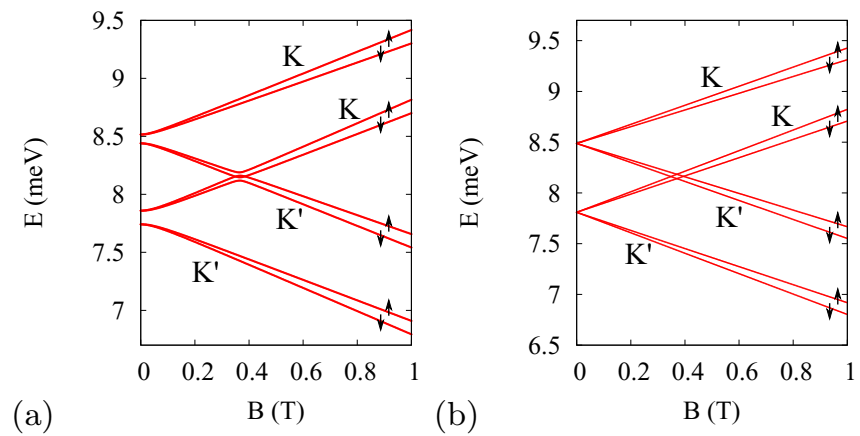

FIG. 5. Eight lowest eigenvalues localized in the dot as a function of perpendicular magnetic field for $2 d=20 \mathrm{~nm}$ as obtained by the tight-binding method. (a) and (b) correspond to smaller and larger flakes, respectively. The avoided crossings in (a) result from the valley mixing by the armchair edge. 


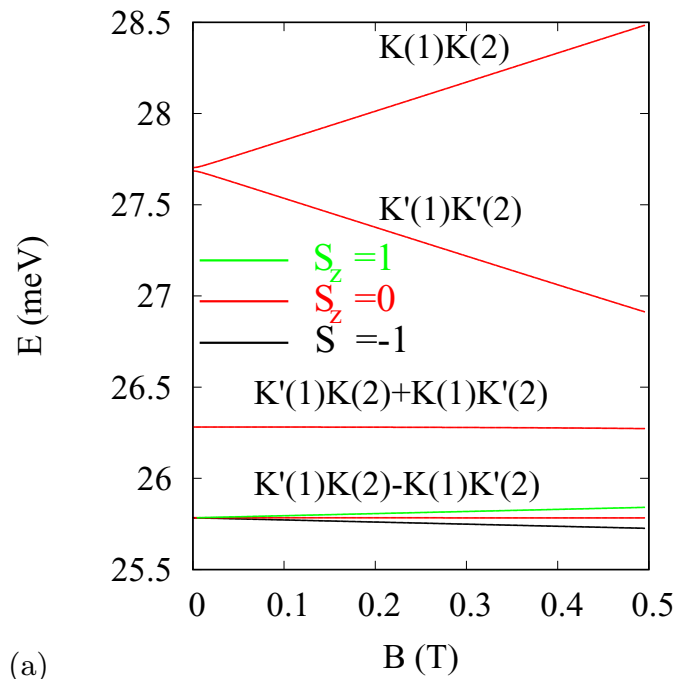

(a)

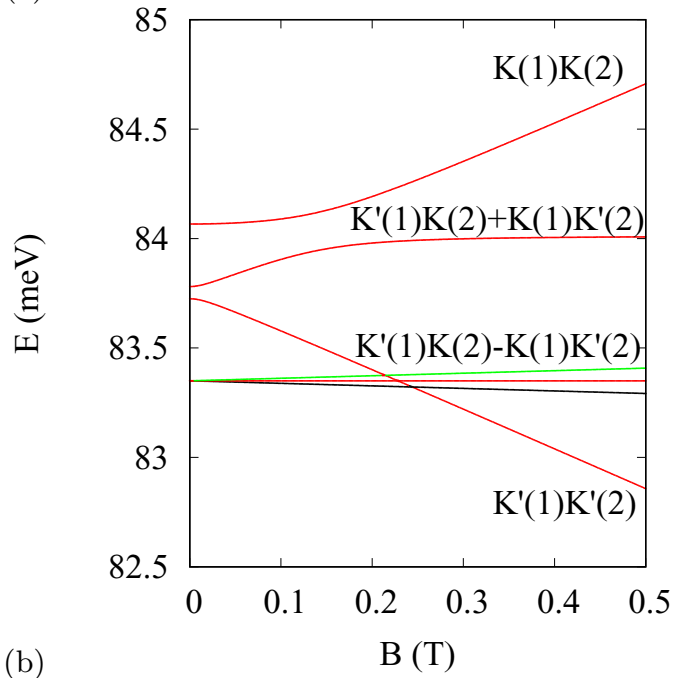

FIG. 6. (a) The low-energy spectrum of the DQD potential for $2 d=6 \mathrm{~nm}$. (b) Same as (a), but for a single QD defined within the flake. Both results are obtained for the smaller flake.

\section{B. Two-electron spectra}

\section{Low-d limit}

The energy spectrum taken for a small interdot distance of $2 d=6 \mathrm{~nm}$ is displayed in Fig. 6(a). At $B=0$ the ground state is threefold degenerate. One of the electrons occupies the $K$ valley, and the other occupies the $K^{\prime}$ valley. The valley degree of freedom allows the electrons to acquire the same spatial distribution with the three possible components of the total spin. From the dominant contributions to the wave function one concludes that the approximate form of the wave function for the threefold-degenerate ground state can be put in the separable form (normalization skipped) $\Psi_{g s}=$ $\psi(1) \psi(2)\left[K(1) K^{\prime}(2)-K^{\prime}(1) K(2)\right] \Psi_{T}$, where $\psi$ is the spatial orbital for the positive $\hat{U}_{P}$ eigenvalue and $\Psi_{T}$ is one of the spintriplet wave functions for $S_{z}=-\hbar, 0$, or $\hbar$. The triplet ground state at $B \neq 0$ is split only by the Zeeman effect. The first excited state is a nondegenerate spin singlet that corresponds to electrons in opposite valleys, $\Psi_{4}=\psi(1) \psi(2)\left[K(1) K^{\prime}(2)+\right.$ $\left.K^{\prime}(1) K(2)\right] \Psi_{S}$, where $\Psi_{S}=\left(\uparrow_{1} \downarrow_{2}-\uparrow_{2} \downarrow_{1}\right)$. The second excited state at $B=0$ is twofold degenerate. Both the electrons occupy either the $K^{\prime}$ valley (the energy level that goes down in $B), \Psi_{5}=\psi(1) \psi(2) K^{\prime}(1) K^{\prime}(2) \Psi_{S}$, or the $K$ valley (the energy that goes up). Still, the spatial orbital is the same for both electrons, which is possible only due to the opposite spins of the states: they are both spin singlets.

For the strong-coupling case in Fig. 6(a) the two dots effectively form a single system, and the spectrum resembles the one calculated for a single dot in Fig. 6(b). The difference for the circular dot is that the valley unpolarized singlet goes higher in the energy at $B=0$. The avoided crossing with the $K K$ energy level as well as the avoided crossing between the two lower singlets at $B=0$ is due to the valley mixing effects of the armchair boundary condition. The ground state is still the triplet, which was previously found for a single-dot study in Ref. [31].

\section{Weak coupling}

For electrostatic quantum dots the coupling between the dots is usually weak, so this case deserves closer inspection as the one which is the most likely to be encountered in an experimental situation. For a weak coupling of $2 d=20 \mathrm{~nm}$ the states can be separated into subgroups with both electrons in separate dots or with both electrons in the same dot. The latter states appear higher in the energy spectrum, and they are characterized by a stronger electron-electron interaction. The right panel in Fig. 7 displays the energy spectrum in a wider energy range as a function of the external field, and the color of the lines displays the average electron-electron interaction energy. In Figs. 7(a) and 7(b) we display a zoom of the spectrum at the energy levels with the electrons in the same dots for the smaller flake and for the larger one, respectively. The spectrum in Fig. 7(a) is very close to the one displayed in Fig. 6(b) for a single dot in a smaller flake. In Fig. 7(b) the spectrum is similar, except the avoided crossing for opposite valleys is closed. The closing of the avoided crossing in the two-electron spectra in Figs. 7(b) and 7(d) (for the larger flake) with respect to Figs. 7(a) and 7(c) (for the small flake) has the same origin as the closing of the avoided crossing of the single-electron spectra in Fig. 5(b) with respect to Fig. 5(a); that is, it results from the removed coupling of the quantum dot confined energy levels with the edge. The considered armchair edge of the flake is not equivalent with respect to $A$ and $B$ and, respectively, the $A$ and $B$ sublattices. The result of this nonequivalence is intervalley mixing of the states coupled to the edge. For a larger distance between the dots and the edge, the valley mixing disappears, and the states of opposite valleys change their order in a crossing instead of an avoided crossing.

The difference between the single QD [Fig. 6(b)] result and the DQD [Fig. 7(a)] is that for the latter, the energy levels shift in pairs. The electron couple can be stored by the left $l$ or the right $r$ dot, and the factor of the spatial wave function can be put in one of the forms $\{l(1) l(2) \pm r(1) r(2)\} / \sqrt{2}$. The tunnel coupling between the dots leads to splitting of the energy levels into bonding and antibonding pairs that are observed in Fig. 7(a).

The ground state of the two-electron spectrum of Fig. 7 corresponds to separated electrons and is displayed in Figs. 7(c) and 7(d) for the smaller and larger flakes, respectively. Here, the two electrons occupy different spatial orbitals, $l$ or $r$. 

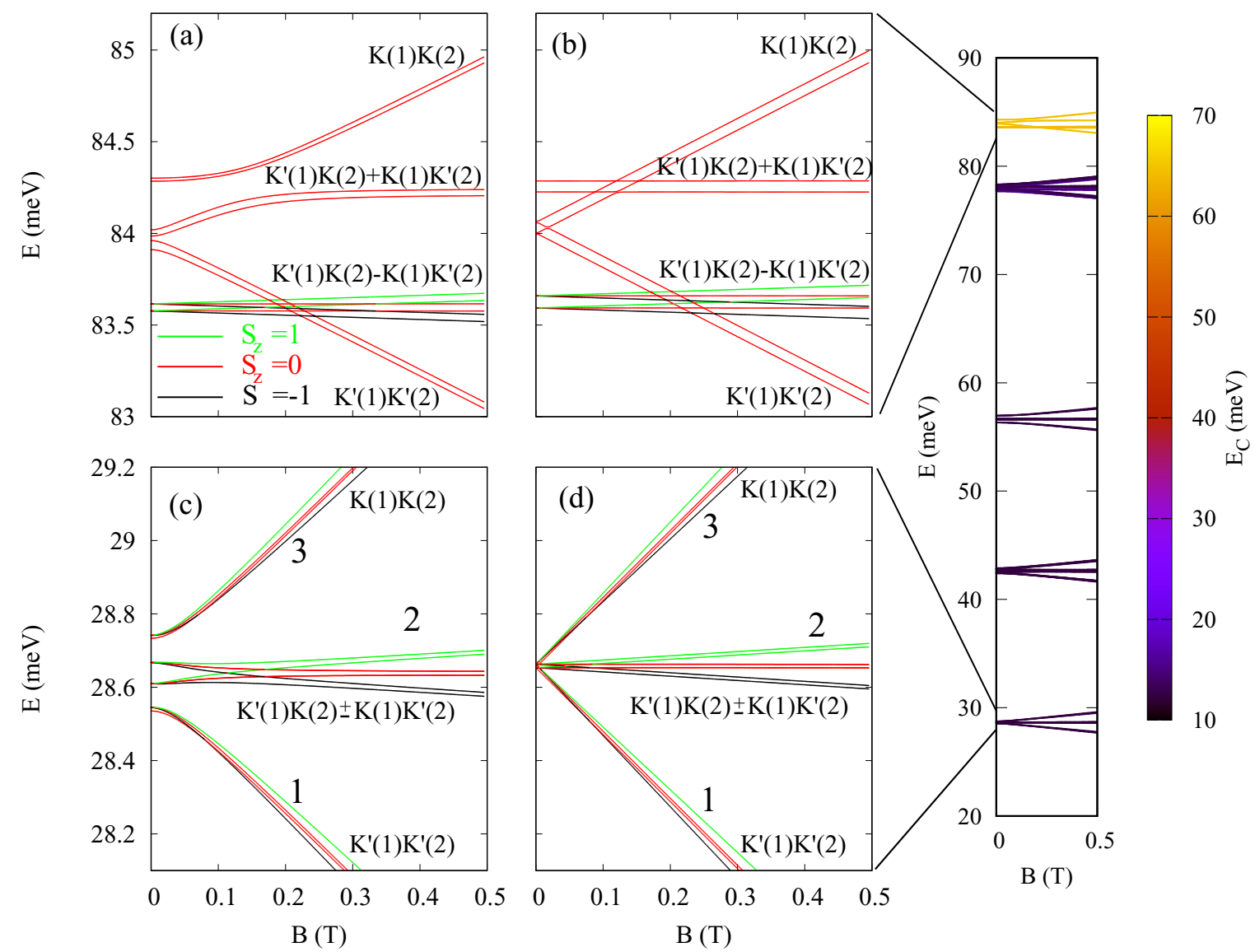

FIG. 7. The two-electron energy spectrum for $2 d=20 \mathrm{~nm}$. The panel on the right shows the groups of energy levels, with the color of the line indicating the expectation value of the electron-electron interaction energy. (a) and (b) Zoom of the high-energy spectrum with electrons in the same dot; (c) and (d) zoom of the ground state. In (a) and (c) the smaller flake was used, while in (b) and (d) the larger one was used for calculations.

For that reason the Pauli exclusion does not forbid them to occupy any of the spin-valley combinations; hence the ground state at $B=0$ is nearly 16 times degenerate for the larger flake [Fig. 7(d)]. For the smaller flake [Fig. 7(c)] at $B=0$ the energy levels split into four quadruples. In the lowest (highest) quadruple both electron levels occupy the lower (higher) energy level: they have four spin states to occupy, hence the number of nearly degenerate states. In both the lowest and highest quadruples a spin singlet has slightly lower energy than the spin triplet.

For the larger flake as well as for the smaller one at higher $B$ the single-electron energy levels shift away from the valley mixing avoided crossing, and the two-electron spectrum forms groups depending on the valley configurations. By analysis of the configuration-interaction (CI) components of the wave function we found that the wave functions at high $B$ are approximately separable into the product of spatial, valley, and spin factors $\Psi(1,2)=\Psi_{s} \Psi_{v} \Psi_{\sigma}$, which are listed in Table I for the energy order that is found in Fig. 7(b) for $B=0.3 \mathrm{~T}$. In the group labeled 1 (3) both electrons occupy the $K^{\prime}(K)$ valley. Each group is formed by four energy levels with the splitting that is due to the Zeeman interaction. Since the valley degree of freedom is frozen, the structure of each of the groups is identical to the one found for two-electron quantum dots in III-V materials, with the spin-singlet $(S)$ and spin-triplet energy level of zero $z$ component of the spin $\left(T_{0}\right)$ split by the exchange energy. Here, the splitting due to the exchange energy is of the order of $9 \mu \mathrm{eV}$.

In the central group of energy levels, labeled 2 in Fig. 7(b), the electrons occupy opposite valleys, hence a nearly constant dependence on $B$, which here is only due to the Zeeman interaction. Each of the $S_{z}=0$ states is twofold degenerate (energy levels $7-10$ in Table I): only the symmetry of the spatial wave function with respect to the interchange of electrons influences the energy of the state via the interdot tunneling effect, and there are two spin and valley factors for both symmetric and antisymmetric states. For each pair of energy levels of group 2 at high $B$ the one that is lower in energy corresponds to a symmetric spatial wave function. The splitting energy is nearly the same as that between the red energy levels of groups 1 and 3. For all the energy levels that shift in pairs in Fig. 7(d) the splitting is due to a difference in the expectation value of the electron-electron interaction energy calculated for symmetric and antisymmetric spatial wave functions, i.e., to the exchange integral [9].

\section{ASYMMETRIC DQD}

For the symmetric system of quantum dots we described above, the splitting of the energy levels is uniquely due to the interdot tunnel coupling. In an experimental situation deviation 
TABLE I. The symmetries of the two-electron states, including the spatial $\left(\Psi_{s}\right)$, the valley $\left(\Psi_{v}\right)$, and the spin $\left(\Psi_{\sigma}\right)$ factors, where the total wave function $\Psi(1,2)=\Psi_{s} \Psi_{v} \Psi_{\sigma}$. This table corresponds to Fig. 7(d), and the first column orders the state in increasing energy order. Levels 7-8 and 9-10 are degenerate. The list corresponds to the dominant contribution to the CI wave functions.

\begin{tabular}{|c|c|c|c|}
\hline Level & $\Psi_{s}$ & $\Psi_{v}$ & $\Psi_{\sigma}$ \\
\hline 16 & $l(1) r(2)-r(1) l(2)$ & $K(1) K(2)$ & $\uparrow(1) \uparrow(2)$ \\
\hline 15 & $l(1) r(2)-r(1) l(2)$ & $K(1) K(2)$ & $\uparrow(1) \downarrow(2)+\downarrow(1) \uparrow(2)$ \\
\hline 14 & $l(1) r(2)+r(1) l(2)$ & $K(1) K(2)$ & $\downarrow(1) \uparrow(2)-\uparrow(1) \downarrow(2)$ \\
\hline 13 & $l(1) r(2)-r(1) l(2)$ & $K(1) K(2)$ & $\downarrow(1) \downarrow(2)$ \\
\hline 12 & $l(1) r(2)-r(1) l(2)$ & $K(1) K^{\prime}(2)+K^{\prime}(1) K(2)$ & $\uparrow(1) \uparrow(2)$ \\
\hline 11 & $l(1) r(2)+r(1) l(2)$ & $K(1) K^{\prime}(2)-K^{\prime}(1) K(2)$ & $\uparrow(1) \uparrow(2)$ \\
\hline $9-10$ & $l(1) r(2)-r(1) l(2)$ & $K(1) K^{\prime}(2)+K^{\prime}(1) K(2)$ & $\downarrow(1) \uparrow(2)+\uparrow(1) \downarrow(2)$ \\
\hline $9-10$ & $l(1) r(2)-r(1) l(2)$ & $K(1) K^{\prime}(2)-K^{\prime}(1) K(2)$ & $\downarrow(1) \uparrow(2)-\uparrow(1) \downarrow(2)$ \\
\hline $7-8$ & $l(1) r(2)+r(1) l(2)$ & $K(1) K^{\prime}(2)-K^{\prime}(1) K(2)$ & $\downarrow(1) \uparrow(2)+\uparrow(1) \downarrow(2)$ \\
\hline $7-8$ & $l(1) r(2)+r(1) l(2)$ & $K(1) K^{\prime}(2)+K^{\prime}(1) K(2)$ & $\downarrow(1) \uparrow(2)-\uparrow(1) \downarrow(2)$ \\
\hline 6 & $l(1) r(2)-r(1) l(2)$ & $K(1) K^{\prime}(2)+K^{\prime}(1) K(2)$ & $\downarrow(1) \downarrow(2)$ \\
\hline 5 & $l(1) r(2)+r(1) l(2)$ & $K(1) K^{\prime}(2)-K^{\prime}(1) K(2)$ & $\downarrow(1) \downarrow(2)$ \\
\hline 4 & $l(1) r(2)-r(1) l(2)$ & $K^{\prime}(1) K^{\prime}(2)$ & $\uparrow(1) \uparrow(2)$ \\
\hline 3 & $l(1) r(2)-r(1) l(2)$ & $K^{\prime}(1) K^{\prime}(2)$ & $\uparrow(1) \downarrow(2)+\downarrow(1) \uparrow(2)$ \\
\hline 2 & $l(1) r(2)+r(1) l(2)$ & $K^{\prime}(1) K^{\prime}(2)$ & $\downarrow(1) \uparrow(2)-\uparrow(1) \downarrow(2)$ \\
\hline 1 & $l(1) r(2)-r(1) l(2)$ & $K^{\prime}(1) K^{\prime}(2)$ & $\downarrow(1) \downarrow(2)$ \\
\hline
\end{tabular}

from the ideal symmetry is inevitable. For a generalization we consider the case of asymmetric quantum dots. The asymmetry is introduced with the confinement potential that includes an in-plane electric field

$$
V_{Q D}(x, y)=-V \exp \left\{-\left[(|x|-d)^{2}+y^{2}\right] / R^{2}\right\}\left(1-\phi \frac{x}{d}\right),
$$

with $V$ and $R$ kept unchanged. The value of the electric field is controlled by a dimensionless $\phi$ parameter, and $d$ in the denominator of the expression in parentheses keeps the potential of the left and right dots in a fixed offset as the interdot distance $2 d$ is varied. The in-plane field in an experimental situation corresponds to a bias between the dots that is introduced to induce the flow of the current.

The single-electron spectrum presented in Fig. 8(a) indicates that the in-plane electric field splits the degeneracy of the energy levels at $B=0$. For large interdot distance [Fig. 8(b)] the single-electron states are localized either in the left or right quantum dot. In the presence of the in-plane
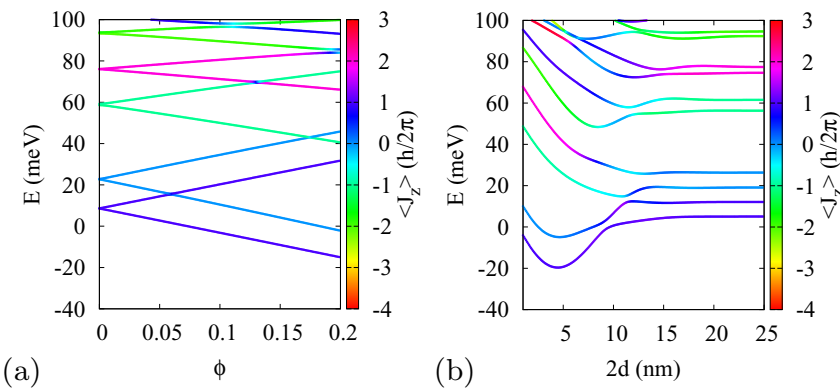

FIG. 8. Single-electron spectrum as a function of (a) the asymmetry parameter $\phi$ for interdot distance $2 d=25 \mathrm{~nm}$ and (b) interdot distance for $\phi=0.03$. Results were obtained with the low-energy continuum approximation. The color of the lines indicates the average value of the total angular momentum operator. field the angular momentum is no longer quantized, but the quantum-mechanical expectation values are preserved in the weak-coupling limit with respect to the symmetric case [compare Figs. 8(b) and 3(c)]. Moreover, the parity symmetry of the external potential is broken by the in-plane electric field. In consequence avoided crossings are opened between the energy levels as the interdot distance is varies [Fig. 8(b)]. Nevertheless, for a small interdot distance the tunnel coupling prevails over the interdot asymmetry, and the results for both the energy levels and the angular momenta are similar to the ideal case [compare Figs. 8(b) and 3(c)].

Figure 9 shows the spectrum in the external magnetic field. The asymmetry of the confinement potential increases the energy spacing between the degenerate doublets at $B=0$, displacing the crossing of the $K$ and $K^{\prime}$ valley energy levels to higher values of the magnetic field (compare with Fig. 5).

For the low-energy states of the two-electron system at $d \simeq 20 \mathrm{~nm}$ the electrons occupy separate dots. The energy

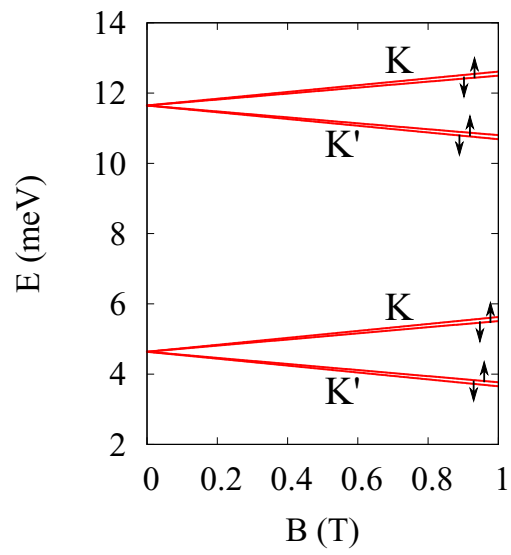

FIG. 9. The same as Fig. 5(b), but for the asymmetric quantum dots with $\phi=0.03$. 
level in one of the dots is shifted up, and the other is shifted down, so no change to the ground-state energy level structure is observed unless the offset of the quantum dot potentials exceeds $60 \mathrm{meV}$, where states with electrons in the same dot occupy the same dot with the energy spectrum presented in Fig. 6(b).

\section{SUMMARY AND CONCLUSIONS}

We have considered the formation of extended orbitals in bilayer graphene quantum dots using atomistic tight-binding and continuum approaches. The various angular momenta in each of the four sublattices for the total angular momentum eigenstates of a circular quantum dot evolve into various spatial parities for each of the wave-function components for the double quantum dot system. The symmetry leads to mixed bonding and antibonding character of wave functions on separate sublattices and a complex dependence of the energy spectrum on the interdot distance. Both a flake large enough to be considered infinite for the dot-localized states and a smaller flake with valley mixing effects of the boundary were described.

For the electron pair we used the configuration-interaction method based on the atomistic single-electron wave functions. We have reproduced the limit result of the spectrum for a single quantum dot when the DQDs are close to one another and identified the effects of the interdot tunnel coupling between the dots for the more realistic weaker interdot tunnel coupling. The effects include the splitting of energy levels in the highenergy part of the spectrum where the electron pair forms bonding and antibonding two-electron orbitals and near the ground-state and the exchange energy that splits the symmetric and antisymmetric pairs of energy levels that shift parallel in the external magnetic field.

\section{ACKNOWLEDGMENTS}

This work was supported by the National Science Centre according to decision DEC-2013/11/B/ST3/03837 and by the Flemish Science Foundation (FWO-VL). Calculations were performed in the PL-Grid Infrastructure.
[1] H. J. Krenner, M. Sabathil, E. C. Clark, A. Kress, D. Schuh, M. Bichler, G. Abstreiter, and J. J. Finley, Phys. Rev. Lett. 94, 057402 (2005).

[2] M. Scheibner, M. Yakes, A. S. Bracker, I. V. Ponomarev, M. F. Doty, C. S. Hellberg, L. J. Whitman, T. L. Reinecke, and D. Gammon, Nat. Phys. 4, 291 (2008).

[3] W. Jaskólski, M. Zieliński, G. W. Bryant, and J. Aizpurua, Phys. Rev. B 74, 195339 (2006).

[4] J. I. Climente, M. Korkusinski, G. Goldoni, and P. Hawrylak, Phys. Rev. B 78, 115323 (2008).

[5] M. F. Doty, J. I. Climente, M. Korkusinski, M. Scheibner, A. S. Bracker, P. Hawrylak, and D. Gammon, Phys. Rev. Lett. 102, 047401 (2009).

[6] C.-Y. Hsieh, R. Cheriton, M. Korkusinski, and P. Hawrylak, Phys. Rev. B 80, 235320 (2009).

[7] R. Hanson, L. P. Kouwenhoven, J. R. Petta, S. Tarucha, and L. M. K. Vandersypen, Rev. Mod. Phys. 79, 1217 (2007).

[8] D. Loss and D. P. DiVincenzo, Phys. Rev. A 57, 120 (1998).

[9] G. Burkard, D. Loss, and D. P. DiVincenzo, Phys. Rev. B 59, 2070 (1999).

[10] I. A. Merkulov, A. L. Efros, and M. Rosen, Phys. Rev. B 65, 205309 (2002).

[11] R. de Sousa and S. Das Sarma, Phys. Rev. B 68, 115322 (2003).

[12] W. Yao, R.-B. Liu, and L. J. Sham, Phys. Rev. B 74, 195301 (2006).

[13] A. V. Khaetskii, D. Loss, and L. Glazman, Phys. Rev. Lett. 88, 186802 (2002).

[14] D. Q. Wang, O. Klochan, J.-T. Hung, D. Culcer, I. Farrer, D. A. Ritchie, and A. R. Hamilton, Nano Lett. 16, 7685 (2016).

[15] H. Bohuslavskyi, D. Kotekar-Patil, R. Maurand, A. Corna, S. Barraud, L. Bourdet, L. Hutin, Y.-M. Niquet, X. Jehl, S. De Franceschi, M. Vinet, and M. Sanquer, Appl. Phys. Lett. 109, 193101 (2016).
[16] B. M. Maune, M. G. Borselli, B. Huang, T. D. Ladd, P. W. Deelman, K. S. Holabird, A. A. Kiselev, I. Alvarado-Rodriguez, R. S. Ross, A. E. Schmitz, M. Sokolich, C. A. Watson, M. F. Gyure, and A. T. Hunter, Nature (London) 481, 344 (2012).

[17] E. A. Laird, F. Kuemmeth, G. A. Steele, K. Grove-Rasmussen, J. Nygård, K. Flensberg, and L. P. Kouwenhoven, Rev. Mod. Phys. 87, 703 (2015).

[18] B. Trauzettel, D. Bulaev, D. Loss, and G. Burkard, Nat. Phys. 3, 192 (2007).

[19] O. Klein, Z. Phys. 53, 157 (1929); M. I. Katsnelson, K. S. Novoselov, and A. K. Geim, Nat. Phys. 2, 620 (2006); J. M. Pereira, Jr., F. M. Peeters, A. Chaves, and G. A. Farias, Semicond. Sci. Technol. 25, 033002 (2010).

[20] M. Y. Han, B. Özyilmaz, Y. B. Zhang, P. Kim, Phys. Rev. Lett. 98, 206805 (2007)

[21] X. L. Liu, D. Hug, and L. M. K. Vandersypen, Nano Lett. 10, 1623 (2010); C.-C. Chen and Y.-C. Chang, Phys. Rev. B 92, 245406 (2015).

[22] C. Stampfer, J. Güttinger, S. Hellmüller, F. Molitor, K. Ensslin, and T. Ihn, Phys. Rev. Lett. 102, 056403 (2009); C. Stampfer, J. Güttinger, F. Molitor, D. Graf, K. Ensslin, and T. Ihn, Appl. Phys. Lett. 92, 012102 (2008).

[23] E. McCann and M. Koshino, Rep. Prog. Phys. 76, 056503 (2013).

[24] E. McCann, Phys. Rev. B 74, 161403(R) (2006); E. V. Castro, K. S. Novoselov, S. V. Morozov, N. M. R. Peres, J. M. B. Lopes dos Santos, J. Nilsson, F. Guinea, A. K. Geim, and A. H. Castro Neto, Phys. Rev. Lett. 99, 216802 (2007); F. Xia, D. B. Farmer, Y. M. Lin, and P. Avouris, Nano. Lett. 10, 715 (2010); J. Park, S. B. Jo, Y.-J. Yu, Y. Kim, J. W. Yang, W. H. Lee, H. H. Kim, B. H. Hong, P. Kim, K. Cho, and K. S. Kim, Adv. Mater. (Weinheim, Ger.) 24, 407 (2012).

[25] J. M. Pereira, Jr., P. Vasilopoulos, and F. M. Peeters, Nano. Lett. 7, 946 (2007). 
[26] L. J. P. Xavier, J. M. Pereira, A. Chaves, G. A. Farias, and F. M. Peeters, Appl. Phys. Lett. 96, 212108 (2010).

[27] S. Döscher, J. Güttinger, T. Mathis, B. Batlogg, T. Ihn, and K. Ensslin, Appl. Phys. Lett. 101, 043107 (2012).

[28] A. M. Goossens, S. C. M. Driessen, T. A. Baart, K. Watanabe, T. Taniguchi, and L. M. K. Vandersypen, Nano. Lett. 12, 4656 (2012).

[29] M. T. Allen, J. Martin, and A. Yacoby, Nat. Commun. 3, 934 (2012).

[30] D. P. Żebrowski, E. Wach, and B. Szafran, Phys. Rev. B 88, 165405 (2013).

[31] M. Zarenia, B. Partoens, T. Chakraborty, and F. M. Peeters, Phys. Rev. B 88, 245432 (2013).

[32] D. P. Żebrowski and B. Szafran, Phys. Rev. B 92, 085307 (2015).

[33] M. Zarenia, A. Chaves, G. A. Farias, and F. M. Peeters, Phys. Rev. B 84, 245403 (2011).
[34] K. G. Wilson, in New Phenomena in Subnuclear Physics, edited by A. Zichichi, Subnuclear Series Vol. 13 (Springer, New York, 1977), pp. 69-142; Y. Tanimura, K. Hagino, and H. Z. Liang, Prog. Theor. Exp. Phys. 2015, 073D01.

[35] A. D. Güclü, P. Potasz, O. Voznyy, M. Korkusinski, and P. Hawrylak, Phys. Rev. Lett. 103, 246805 (2009).

[36] E. N. Osika and B. Szafran, Phys. Rev. B 93, 165304 (2016).

[37] R. Egger and A. O. Gogolin, Phys. Rev. Lett. 79, 5082 (1997).

[38] L. Mayrhofer and M. Grifoni, Eur. Phys. J. B 63, 43 (2008).

[39] A. Secchi and M. Rontani, Phys. Rev. B 88, 125403 (2013).

[40] D. A. Siegel, C.-H. Park, C. Hwang, J. Deslippe, A. V. Fedorov, S. G. Louie, and A. Lanzara, Proc. Natl. Acad. Sci. USA 108, 11365 (2011).

[41] S. Schulz, S. Schumacher, and G. Czycholl, Phys. Rev. B 73, 245327 (2006). 